\title{
Rede, gehalten bei der Eröffnung des XXVI. Kongresses für innere Medizin in Wiesbaden, am 19. April 1909.
}

\author{
Von F. Schultze.
}

M. H.! Wenn man die Reden durchgeht, welche die früheren Vorsitzenden dieses Kongresses gehalten haben, so sind es vor allen Dingen die Gedanken der Autonomie und der Einheitlichkeit der inneren Medizin, die seit der unvergeßlichen ersten Rede von Frerichs immer wieder von neuem betont wurden.

Würde das nötig gewesen sein, so fragt man sich unwillkürlich, wenn nicht das Reich der inneren Medizin eine verhängnisvolle Aehnlichkeit mit dem alten römischen Reiche deutscher Nation gezeigt hätte oder gar noch zeigt, mit jenem Reiche, das so oft von außen her regiert wurde und von dem sich immer mehr einzelne Teile ablösten, bis es endlich völlig zerfiel?

Wie steht es heute mit dieser unserer Autonomie und mit unserer Einheitlichkeit?

Die erstere scheint mir zurzeit unbestritten, während zur Zeit der Frerichsschen Rede Vielen besonders die pathologische Anatomie eine viel zu große Rolle zu spielen schien. Ich sage "schien". Denn in Wahrheit war die Gefahr nicht groß. Auch damals galt den Aerzten und den meisten inneren Klinikern die Förderung der Therapie des kranken Menschen als das höchste Ziel, und nicht in erster Linie die pathologisch-anatomische Diagnose. Ich brauche nur an Brand (Stettin), an Liebermeister, Ziemssen, Jürgensen, Kussmaul und an Friedreich zu erinnern. Obgleich der Letztgenannte direkter Nachfolger Virch ow's in Würzburg war und gewiß die pathologische Anatomie sehr hoch schätzte und pflegte, war er doch vor allem ein Arzt und ein Helfer im besten Sinne des Wortes.

Zurzeit wird die pathologische Anatomie von Manchem eher zu gering eingeschätzt, trotzdem sie doch allein vor Fehldiagnosen 
schützen kann und trotzdem ohne sie so manche Aerzte, die kaum mehr eine Sektion sehen, in ein Labyrinth phantasievoller falscher und unvollständiger Diagnose gelangen und sich infolgedessen allmählich mehr und mehr der rein äußerlichen, symptomatischen Krankheitsauffassung und der rein schematischen Krankheitsbehandlung der Naturheilkünstler in verhängnisvoller Weise annähern.

Glücklicherweise hat gegen früher die pathologische Anatomie am Lebenden gegenüber derjenigen an der Leiche einen ausgedehnteren Boden gewonnen, vor allem durch die Röntgenologie und sodann durch die Autoskopie der Chirurgen, durch welche z. B. erst die gewaltige Bedeutung der Erkrankung des Wurmfortsatzes erkannt wurde, die der Leichenanatomie entgangen war. Eher könnte man heutzutage von einem erneuten Ansturm der Physiologie sprechen, der an sich berechtigter ist, wie der seinerzeit von Rose und Wunderlich unternommene, als sie ihr Archiv für physiologische Heilkunde gründeten.

Denn es ist in neuerer Zeit eine große Fülle neuer physikalischer und chemischer Untersuchungsmethoden zu den alten hinzugetreten und hat die Diagnostik, besonders auch in bezug auf die Funktionsstörungen der Organe erheblich gefördert und der Therapie gute Dienste geleistet.

Da aber auch die Physiologie des gesunden und kranken Menschen geradeso wie die pathologische Anatomie in den Dienst der Beobachtung und Behandlung der Kranken gestellt wird, und da wohl kaum jemand ernstlich daran denkt, die Krankensäle zur Appendix der Laboratorien zu machen, so kann auch aus der so erfreulichen Fortentwicklung der Physiologie des Menschen der inneren Medizin ein Schaden nicht erwachsen und eine Unterordnung der inneren Medizin nicht entstehen.

Viel bedenklicher steht es mit der Aufrechterhaltung der Einheitlichkeit der inneren Medizin.

Die Worte, welche Friedrich Müller über diese so wichtige Sache auf dem vorigen Kongresse gesprochen hat, fanden wohl bei uns allen den größten Anklang, und es kann ihnen heute kaum etwas Neues hinzugefügt werden.

Niemand wird verhindern wollen oder gar verhindern können, da $B$ sich ein klinischer Forscher spezialisiert, zeitweilig oder dauernd - er sammle still und unerschlafft im kleinsten Punkt die größte Kraft - und es haben auch die bedeutendsten Kliniker schon vor Jahrzehnten nicht gleich Großes auf allen Gebieten der inneren Medizin geleistet, nicht gleich Großes z. B. auf den Hauptgebieten der Chemie oder Physik oder auf dem Gebiete der Pathologie und Therapie aller großen einzelnen Krankheitsgruppen.

Niemand kann und wird auch dagegen sein, daß spezialistische Praktiker echter Art nur bestimmte Teile der inneren Medizin pflegen und ausüben, im Gegenteil.

Aber es muB nun einmal allgemeine A erzte geben, Hausärzte und besonders auch Aerzte auf dem Lande, an denen es leider in manchen Gegenden mangelt, wie denn, nebenbei gesagt, die vielbeklagte Ueberfüllung des ärztlichen Berufes keine gleichmäßige ist und sowohl unserem Heere als unseren Kolonien nicht hinreichend viele Aerzte zur Verfügung stehen.

Es muB, nach einem Ausdruck von $\mathrm{Na}$ a nyn, gegenüber den Spezialisten Nothelfer geben, wiewohl doch auch die Spezialisten oft genug Nothelfer sind, und somit kein durchgreifender Unterschied zwischen beiden Arten von Aerzten besteht. Na u n y n braucht den Ausdruck Nothelfer für diejenigen Aerzte, die dann eintreten, wenn es sich um unentwickelte, beginnende Krankheiten handelt, oder um solche, für die wir ein eigentliches Heilmittel noch nicht haben, während der Spezialist sich auf dasjenige beschränken und zurückziehen könne, was wir heute wissen und können.

Um mindestens solche $\mathrm{Nauny}$ sche Nothelfer auszubilden und um vor allem allgemeine Aerzte zu erziehen, muß der angehende $\mathrm{Me}$ diziner die allgemeine Pathologie, die allgemeine Diagnostik und Therapie und ihre Grundsätze kennen lernen und zu ihrer Anwendung befähigt werden.

Es muß somit bei dem Unterrichte in der Medizin auf der Universität Männer geben, die besonders innerhalb derjenigen Fä.cher, welche mit allen Organen des Körpers zu tun haben, wie die innere Medizin, die Chirurgie, oder wenigstens mit einem solchen, das den ganzen Organismus beherrscht, wie das Gehirn, Allgemeinärzte erziehen und auch auf die künftigen Spezialärzte so einwirken. daß diese wenigstens einmal in ihrem Leben gründlich mit den allgemeinen Grundlagen der Pathologie, der Diagnose und Therapie auf dem Gesamtgebiete der Medizin vertraut gemacht wurden.

Nun ist es ja selbstverständlich, daß der innere Kliniker, um hier nur von ihm zu reden, schon lange nicht mehr jeden einzelnen Aufsatz auf seinem Gesamtgebiet oder auch nur jedes Referat von A bis $Z$ lesen kann - das wäre eine Qual der Hölle - und ebenso kann er nicht jede neue Untersuchungs- und Behandlungsmethode und ihre Modifikationen sich bis ins Einzelne zu eigen machen - auch das geht über menschliche Kräfte -, aber er muB sich mit geeigneten Hilfskräften umgeben, die mit ihm zusammen gewissermaßen mit verteilten Rollen auf dem großen Gesamtgebiete arbeiten und auf diese Weise - abgesehen von seinem eigenen Schaffen in allem Wesentlichen theoretisch und praktisch orientiert bleiben.

Kommt ein solcher Zentralkliniker in Wegfall, wohin so Manche streben, gibt es nur noch besondere Einzelkliniker etwa für die Infektionskrankheiten, Stoff wechselkrankheiten, Herzkrankheiten, Nervenkrankheiten, so wird weder ein eigentlicher Kliniker noch ein Arzt mehr erzogen; jeder Lehrende zöge seinen Stoff ins Breite, es gäbe übermäßige Wiederholungen, und es bliebe auf dem Gebiete der inneren Medizin höchstens noch der Pädiater ein Allgemeinkliniker, solange es ihm noch gestattet sein würde, alle inneren Krankheiten vorzustellen und über sie vorzutragen. Was ihm aber recht ist, sollte auch dem inneren Kliniker für die Erwachsenen billig sein.

Nun droht ja eine so weitgehende Zersplitterung der inneren Klinik zurzeit noch nicht - bis auf die Lostrennung der Nervenkrankheiten - die, wenn einmal geschehen, allmählich zu einem höchst beklagenswerten Ausschlusse sämtlicher Nervenkranken aus der inneren Klinik führen würde, also von Kranken, die ihrerseits ganz besonders einer gründlichen Untersuchung aller inneren Organe bedürfen und nicht bloB derjenigen des Nervenapparates.

Aber es droht eine Einschränkung des Unterrichtes in den umfangreicheren Fächern und besonders in der inneren Medizin dadurch, daß ein ausgedehnter theoretischer und prak tischer Unterricht in der sogenannten sozialen Medizin gefordert wird. Sie droht zu einer Zeit, in der zugleich alle Einzelfächer der inneren Medizin, die Pädiatrie mit eingeschlossen, in ihrer Diagnostik und Heilmethodik eine immer größere Ausbildung und Vertiefung erlangt haben. Ich erinnere nur an die so überaus wichtige Säuglingsernährung, an die Behandlung der Säuglingskrankheiten, an die feinere Ausbildung der Untersuchungsmethoden der Funktionen des Herzens und der Gefäße, an die Röntgenologie, an die Bakteriologie, an die physikalischen Heilmethoden und an die Immunodiagnostik, auf chirurgischem Gebiete an die Orthopädie, Urologie etc.

Daß besonders die neue Unfalls- und Invaliditätsgesetzgebung Anforderungen stellte, die nicht sogleich in vollem Maße erfüllt werden konnten, war von vornherein klar und zeigte sich bald. Es mußte erst eine Unfallspathologie und Prognostik zum guten Teile geschaffen werden. Es mußten die Aerzte zu den verlangten genaueren Diagnosen auch von Krankheiten erst erzogen werden, die zu diagnostizieren früher als eine überflüssige Finesse galt.

Weiterhin erscheint es aber den Aerzten ${ }^{1}$ ) nötig, daß außer in der sozialen Versicherungsmedizin auch noch ein besonderer Universitätsunterricht über gewerblichen Arbeiterschutz, kommunalärztliche Tätigkeit, Fürsorgewesen und endlich über Aufbau und Wesen des Aerztestandes und die sozialen Angelegenheiten des Aerztestandes stattfinde.

Der bisherige Unterricht in diesen Dingen in der Hygiene, in der Staatsarzneikunde, in den einzelnen, schon längst an vielen Universitäten gehaltenen Kursen über Versicherungsmedizin mit Krankenbegutachtungen genüge nicht, sondern es müßten neue sozialmedizinische Professuren im Hauptamte, womöglich Ordinariate geschaffen werden, „mit allen theoretischen und praktischen Forschungs- und Unterrichtsmitteln" ausgestattet, also doch mit Instituten. Am geeignetsten seien zu solchen Lehrern ärztliche Praktiker; besondere stationäre klinische Abteilungen seien nicht nötig, die Leiter der klinischen Universitätsinstitute seien aber anzuweisen“, den betreffenden Lehrern stationäre Fälle zu Unterrichtszwecken zur Verfügung zu stellen. Die Studenten seien zum Besuche der Vorlesungen anzuhalten“. So lauten die Hauptforderungen.

Es wird also hier das Umgekehrte gewünscht, wie früher in der Forderung der Vervollkommnung der ärztlichen Ausbildung in der ärztlichen Technik. Diese sollte mehr außerhalb des Rahmens der Universität stattfinden, die sozialmedizinische dagegen hauptsächlich innerhalb desselben.

So sehr nun dieses Vertrauen die Universitäten ehrt, wenn auch die Ergänzung ihrer Lehrkräfte für den Unterricht in der sozialen Medizin durch außenstehende Praktiker für notwendig erachtet wird, so fragt es sich, ob es zweckmäßig ist, in die Zahl der

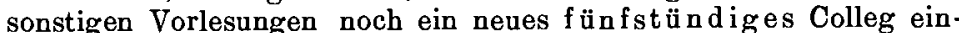
zuschieben, wie das verlangt wird.

Jeder Universitätslehrer, der weiß, wie zurzeit eine ganze Reihe wichtiger, für die Ausübung der ärztlichen Praxis absolut nötiger Kurse kaum untergebracht werden kann, jeder, der weiß, daß schon jetzt die wichtigen Vorlesungen über spezielle Pathologie

1) Die soziale Medizin als Gegenstand des Unterrichtes, von Dr. Alf red Pey se r (Berlin). Denkschrift, verfabt im Auftrage des Geschäftsausschusses des Deutschen Aerztevereinsbundes. 
wegfallen, obwohl in ihnen manches auf die soziale Medizin Bezügliche beigebracht werden kann, jeder, der weiß, daß schon jetzt bei der Abneigung der Studierenden, theoretische Collegs zu hören, über die Abnahme des Interesses für so wichtige Fächer wie allgemeine Pathologie und pathologische Anatomie geklagt wird, wird sich sagen müssen, daß ein so ausgedehntes Colleg wie das erwähnte, wenn überhaupt, so nur mit Hilfe von nicht recht geeigneten Mittags- oder Abendstunden untergebracht werden kann.

Man müßte denn die Ferien bedeutend kürzen, was für die Studenten vielleicht durchführbar wäre, wenn auch nicht gut für die Mediziner allein, aber nicht für die meisten klinischen Lehrer, deren Ferienzeit jetzt durch Examina und durch Fortbildungskurse in ganz anderer Weise als früher in Anspruch genommen wird, sodaß die Zeit für die eigene Forschungs- und Schaffenstätigkeit sowie für eine Erholung immer kürzer geworden ist.

Oder man $\mathrm{mu} \beta$ auf das praktische Jahr rekurrieren, dem diese Aufgabe von vornherein mit vollem Rechte zugedacht war. Denn in ihm hat der angehende Arzt praktisch mit dem Krankenkassenwesen, mit den Standesinteressen, mit der Organisation der Aerzte zu tun. Und erst dadurch wird der junge Mediziner naturgemäß das wahre Interesse an den Aufgaben der sozialen Medizin gewinnen, daß er mit ihnen praktisch in Berührung kommt, gerade so wie er nicht durch theoretische Vorlesungen ïber spezielle Pathologie mit oder ohne Lichtbilder, sondern erst durch die Kurse und durch die Klinik zu einem inneren Anteil an der Pathologie und Therapie gebracht wird.

Und ebenso wäre hier ein Feld für die Betätigung der allerdings an Zahl geringen Akademien für praktische Medizin gegeben, die ja mit zu diesem Zwecke gegründet sind, zu denen aber freilich zurzeit die Medizinalpraktikanten noch viel weniger hinströmen als selbst zu den Universitätsinstituten, zum guten Teile aus dem Grunde, weil Städte und Staat bisher davor zurückschreckten, den Praktikanten solche Vorteile zu bieten wie viele kleinere Krankenhäuser, denen oft kein so großes Lehrmaterial zur Verfugung steht als jenen.

Preßt man aber trotz alledem die gesamte soziale Medizin mit Gewalt in den jetzigen Unterricht hinein, dessen Zeit um ein weiteres Semester zu verlängern doch auch wieder manche Nachteile hätte, so gereicht das wohl nach der Meinung der meisten Universitätslehrer, die doch in bezug auf die Leistungen bei den Prufungen die gegebenen Beurteiler sind, vielleicht der Quantität, aber nicht der Qualität des medizinischen Unterrichtes zum Vorteil.

Denn auch jetzt darf man im Examen die Anforderungen nicht hoch stellen, wenn auch nach Wegfall der Prüfung in der Physiologie und der deskriptiven Anatomie mir eine entschiedene Besserung eingetreten zu sein scheint. Der Lücken sind oft noch allzuviele.

Endlich spricht gegen die allzuweite Ausdehnung des Unterrichts in der sozialen Medizin, und un diese handelt es sich, nicht um den Unterricht dieser Art überhaupt, der Umstand, daß es dem betreffenden neuen Lehrer dieses Faches doch niemals möglich sein wird, in allen Teilen der Medizin zugleich ein hinreichend kompetenter Obergutachter und damit Lehrer zu sein, etwa zugleich nur in der Chirurgie und in der inneren Medizin, geschweige denn in der Psychiatrie, Augenheilkunde etc. Es muß also doch, trotz der Existenz eines besonderen sozialmedizinischen Professors im Hauptamt entweder ein besonderer Kurs wenigstens in den beiden Hauptfächern für die Unfall- und Invaliditätsmedizin abgehalten werden, wie vielfach bisher, wodurch von neuem Zeit beansprucht wird, oder in den kleinen Fächern von dem jeweiligen Lehrer besonders auf diese Dinge Gewicht gelegt und darüber Vortrag gehalten werden.

Unbedingt muß aber daran festgehalten werden, daß diejenigen am besten für die spätere Gutachtertätigkeit vorbereitet sein werden, die am besten mit der Diagnostik und mit der gesamten allgemeinen und speziellen Pathologie und Therapie, und zwar besonders der Aetiologie und Prognostik vertraut sind, also mit denjenigen Fächern, die durch ausgedehnte neue Collegs eine Einbuße erfahren.

Der Universitätsunterricht kann überall nur die Grundlagen für das ärztliche Wissen und Können legen, vor allem die Methodik lehren und die Ehrfurcht vor der wissenschaftlichen Forschung. Er soll auch vor allem seine Adepten mit dem Geiste wissenschaftlicher Kritik durchdringen.

Denn eine solche Kritik ist heute nötiger als je. Sie ist mehr als früher erforderlich für die Tätigkeit des künftigen Gutachters, die so ungemein erziehlich wirkt. Sie ist mehr als je erforderlich in der Therapie, die mit einer Flut von Heilmitteln und Heilmethoden den Arzt überschwemmt und nur zu leicht den Wert und die Bedeutung der seelischen Einwirkung, der Suggestion übersehen und unterschätzen läßt.

Der Universitätsunterricht ist endlich in Deutschland aufgebaut auf dem Grundsatze der akademischen Freiheit und widerstrebt mit Recht den Forderungen allzuvieler Reglementierungen und $Z$ wangsmaßregeln, die nur scheinbar nützen. Gerade so wie wir alle wünschen, daß der ärztliche Beruf ein möglichst freier bleiben möge, so wünschen auch die vielgescholtenen Fakultäten ein Festhalten an der akademischen Lehr- und Lernfreiheit, unter deren Wirkung sich die deutschen Aerzte ihre angesehene Stellung in der Welt errungen haben. 